\title{
Thin-blade penetration resistance and snow strength
}

\author{
C.P. BORSTAD, ${ }^{1}$ D.M. MCCLUNG ${ }^{2}$ \\ ${ }^{1}$ Department of Civil Engineering, University of British Columbia, 6250 Applied Science Lane, Vancouver, \\ British Columbia V6T 1Z4, Canada \\ E-mail: cborstad@civil.ubc.ca \\ ${ }^{2}$ Department of Geography, University of British Columbia, 1984 West Mall, Vancouver, British Columbia V6T 1Z2, Canada
}

\begin{abstract}
A thin-blade snow hardness gauge was developed that measures penetration resistance over a length scale (on the order of 10-100 grain contacts) relevant to the fracture of slab avalanches. A thin blade was chosen to measure the ruptures of bonds and grain structures and minimize the effects of snow compaction during penetration. The apparatus consists of a $10 \mathrm{~cm}$ wide, $0.6 \mathrm{~mm}$ thick stainlesssteel blade attached to a digital push-pull gauge. Blade penetration measurements are easy to conduct in the field and laboratory and required no post-processing or subjective interpretation. Measurements were conducted in snow pits to test the effects of penetration rate, blade orientation and blade width. The blade hardness index, defined as the maximum force of penetration, is a highly repeatable measure across observers compared to the hand hardness test. The blade hardness index was a better variable than the density for correlating with tensile strength measurements in a cold laboratory and with a cohesive strength measure in the field. As strength is one of the most important parameters in the fracture mechanics of slab avalanches, the strong correlation between thin-blade penetration and strength should benefit future slope stability evaluations using this gauge.
\end{abstract}

\section{INTRODUCTION}

Snow hardness is defined as the resistance to penetration of an object into snow (Fierz and others, 2009) and is measured using penetrating devices of various shapes and sizes. The resisting force in any hardness measure comes from a combination of bending and rupture of grain bonds and grain structures, compaction of loose grains and friction between snow and the penetrating object. The relative contribution of each resistance component to the total penetration force is unknown. However, bonding is the critical variable in determining the mechanical properties of snow such as strength (Shapiro and others, 1997).

Despite the recognition of the relationship between strength or bonding in snow and hardness, the bulk density of snow continues to be the most commonly used index variable for mechanical properties of snow. Examples of properties represented as functions of density include strength and Young's modulus (e.g. Shapiro and others, 1997; Marshall and Johnson, 2009; Nakamura and others, 2010), fracture toughness (Sigrist and others, 2005), fracture speeds and fracture energy (McClung, 2007a,b) and viscoelastic properties (Camponovo and Schweizer, 2001). The scatter in properties at a given density is typically attributed to differences in snow microstructure (Schweizer and others, 2003).

Several factors explain the widespread use of density in these contexts, when a hardness measure or other parameter representing bonding is theoretically more appropriate. Density is easy to measure and relatively objective, though different density samplers can give rise to inconsistent results with different errors (Conger and McClung, 2009). More importantly, no objective standard for hardness has been adopted to supplement or replace the density as a proxy variable in snow mechanics.

A thin-blade snow hardness gauge was developed to establish an objective index measure of hardness for direct comparison with strength and other mechanical properties of snow. In order to minimize compaction and displacement of snow ahead of the penetrating tip, the thickness of the blade $(0.6 \mathrm{~mm}$ at the leading edge) was chosen to be comparable to the grain sizes commonly encountered in alpine snow. The width of the blade $(10 \mathrm{~cm})$ was chosen so that around 10-100 grains would be simultaneously in contact with the blade, resulting in an average resistance measure over a length scale that corresponds to the structural scale of interest in most avalanche applications. Examples of relevant length scales in fracture of snow include the critical length of weak-layer fractures (often called sweet spots or hot spots) prior to unstable propagation, which are on the order of the slab depth (Bažant and others, 2003), and the scale of distributed damage prior to tensile crack coalescence, which is on the order of 10-100 times the grain size (Borstad and McClung, http:// www.geog.ubc.ca/avalanche/pubs/BorstadMcClung_2009_ ICF12.pdf).

The blade hardness gauge consists of an adapted stainless-steel paint-scraper blade attached to a hand-held push-pull gauge. The peak resistance to penetration of the blade into layers or samples of snow is defined as the blade hardness index and is the single quantitative output. Blade hardness measurements were made horizontally in the walls of excavated snow pits. The results were compared against hundreds of density and hand hardness tests. The effects of penetration rate, blade orientation and blade width were explored. The blade hardness index was a consistent measure across observers, overcoming a drawback of the common hand hardness test. The tensile strength of snow samples was measured in a cold laboratory, and the strength correlated better with the blade hardness index than with the density. A threshold in penetration resistance was identified that separated cohesive from cohesionless snow, confirming previous results using a thin-blade gauge (Fukue, 1977).

The scope of the present investigation was limited to an exploration of the blade hardness gauge with respect to its response in different types of snow and environmental conditions, sensitivity to various testing conditions and 


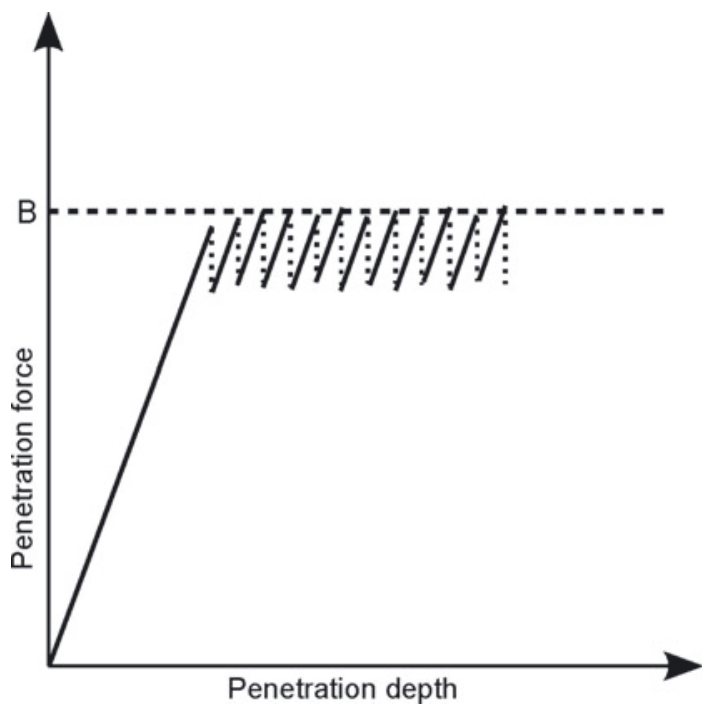

Fig. 1. Conceptual schematic (not to scale) of blade penetration force versus penetration distance for brittle penetration rates, based on measurements made by Fukue (1977). In Fukue's study, the minima following individual peaks in force were located at less than half of the peak force. The wider blade in this study should lead to higher minima with respect to individual peaks due to more structural elements in contact with the blade. The blade hardness index, $B$, is represented by the dashed line.

usefulness in providing a single quantitative output for correlation with strength and other mechanical properties of snow related to avalanches. Correlation and comparison with common instability evaluation tests in avalanche work is beyond the scope of the analysis but will be an important area of future research.

We begin with a brief review of relevant hardness literature. Emphasis is given to results related to thin-blade penetration, compaction of snow in hardness measures and comparisons between direct measurements of strength and hardness. Details of the design and use of the thin-blade gauge follow. Tests involving the gauge in excavated snow pits and in the cold laboratory are described, followed by the results of these tests and discussion. Potential applications of the gauge and limitations of the present study are discussed and conclusions drawn.

\section{HARDNESS MEASURES}

\subsection{Thin-blade hardness}

Bradley (1966) developed a resistograph that recorded hardness using two blades mounted on either side of a probe. The probe was inserted to the base of the snow, rotated by $90^{\circ}$ and withdrawn. The resisting force met by the blades during withdrawal was transferred via a spring in the shaft to a scribe that recorded the force on a spool of paper. This design was later modified so that the resistance was met by two upward-pointing cones rather than blades (Bradley, 1968). This change may have been the result of difficulty in turning the blades prior to withdrawal in some types of snow (Floyer, 2008). Bradley's resistograph was never widely adopted.

Fukue (1977) carried out thin-blade penetration measurements that met four primary objectives: (1) the measure was simple to carry out, (2) it minimized sensitivity to penetration rate, (3) it minimized densification of snow around the penetrating object and (4) it minimized changes in intergranular bonding between adjacent snow grains. The third point has been emphasized as a drawback of many common hardness measures (Shapiro and others, 1997). The last two points may be especially important in any hardness measure with a large compaction zone since ice grains form bonds which gain strength within a fraction of a second after contact (Szabo and Schneebeli, 2007).

The blade used by Fukue (1977) was $12 \mathrm{~mm}$ wide and $0.6 \mathrm{~mm}$ thick, with a blunt leading edge. It was mounted to an actuator and driven into snow samples in a cold laboratory, and the penetrating force was measured using a transducer. The individual peaks in the force-depth signal were roughly constant within the first $3 \mathrm{~cm}$ of penetration depth and slightly increased with further penetration due to friction between the sides of the blade and the snow grains. A ductile-to-brittle transition in penetration speed of $0.2 \mathrm{~mm}$ $\mathrm{s}^{-1}$ was observed. At penetration speeds below this transition, the response of the snow was ductile, characterized by penetrating force which increased without bound. At speeds above this transition, brittle bond failures were evident from the spiked shape of the force displacement signal (Fig. 1). A slight rate dependence in the brittle range, with decreasing peak penetration force with increasing penetration speed, was observed between 0.2 and $0.6 \mathrm{~mm} \mathrm{~s}^{-1}$. Above $0.6 \mathrm{~mm} \mathrm{~s}^{-1}$ the peak force was independent of penetration speed.

Similar trends in the vicinity of the ductile-to-brittle transition were also observed in the uniaxial tensile strength tests, expressed as a function of strain rate, reported by Narita (1980). The maximum blade penetration force in Fukue's data and the maximum tensile strength in Narita's data, both as functions of rate (penetration rate and strain rate, respectively), were observed at the ductile-to-brittle transition. This suggests that Fukue's ductile-to-brittle transition at a penetration speed of $0.2 \mathrm{~mm} \mathrm{~s}^{-1}$ corresponds to a bond-scale strain rate on the order of $10^{-4} \mathrm{~s}^{-1}$.

\subsection{Hand hardness}

The hand hardness test (de Quervain, 1951) is perhaps the most common hardness test in avalanche-forecasting work (McClung and Schaerer, 2006) and is, consequently, commonly cited in analysis of avalanche and snow stability data (e.g. Schweizer and Jamieson, 2001, 2007). Its basic premise is to penetrate the snow using a standard force. Achieving this standard force requires selecting penetrating objects of different cross-sectional area. Five hand hardness categories (excluding solid ice) are defined corresponding to different cross-sectional areas that can be driven into the snow layer without exceeding the given force (Fierz and others, 2009). Operationally, half-scale or \pm qualifiers are often appended to the categorical result to refine the coarse scale.

The current international standard penetration force in the hand hardness test is $10-15 \mathrm{~N}$ (Fierz and others, 2009). The previous version of the hardness standard (Colbeck and others, 1990) specified a force of $50 \mathrm{~N}$. However, the North American standard has been $10-15 \mathrm{~N}$ for many years (McClung and Schaerer, 2006). When comparing different hand hardness indices from different observers, countries or years, therefore, the difference in the applied force may vary by up to a factor of five. This makes any quantitative analysis using the hand hardness index difficult.

Höller and Fromm (2010) measured actual force values associated with the hand hardness test using a push-pull 
gauge and flat plates with standard cross-sectional areas. Their results showed high scatter and overlap between penetration resistance values for adjacent hand hardness categories. Overall, the maximum force values agreed better with the old $50 \mathrm{~N}$ force standard (Colbeck and others, 1990), though the force gauge was unable to record values below $10 \mathrm{~N}$. These results illustrate the limitations to quantitative analysis using hand hardness data.

\subsection{Probe hardness}

The Swiss rammsonde or ram hardness test (Bader and others, 1954) is a cone penetration test $\left(60^{\circ}\right.$ cone tip angle and $40 \mathrm{~mm}$ base diameter) adapted from the soil sciences. The ram resistance is defined as the measured amount of force required to drive the rod a given depth into the snow. The large base area and weight of the instrument limit the vertical resolution of the ram hardness to the centimeter scale (Pielmeier and Schneebeli, 2003).

The SnowMicroPen (SMP) is a motor-driven cone penetrometer that records hardness at sub-millimeter resolution (Schneebeli and Johnson, 1998; Johnson and Schneebeli, 1999). The cone angle is the same as the Swiss rammsonde, but the cone diameter $(5 \mathrm{~mm})$ is much smaller. Pielmeier and Schneebeli (2003) compared SMP hardness profiles to hand hardness and ram hardness and found that the SMP most effectively resolved small-scale stratigraphy when compared against planar sections of snow layers. No standard algorithm exists for interpreting and processing the SMP resistance signal, making comparison of results from different studies difficult (Marshall and Johnson, 2009). The SABRE probe penetrometer (Mackenzie and Payten, 2002) is another probe hardness gauge, with a $12 \mathrm{~mm}$ diameter rounded tip, that has seen limited use (Floyer, 2008).

\subsection{Compaction of snow in hardness measures}

Floyer (2008) attached tips of different shape and size to the SABRE probe, filmed the penetration pattern around each, and analyzed the films using particle-tracking velocimetry. Floyer and Jamieson (2010) examined in more detail the compaction around the round probe tip specifically. These experiments provide insight into the assumption that compaction around a probe tip can be neglected in the interpretation of the force signal (e.g. Johnson and Schneebeli, 1999; Marshall and Johnson, 2009). This compaction can be separated into horizontal (or normal to the direction of penetration) and forward (ahead of the probe tip) components.

The most important qualitative conclusion that can be drawn from the work of Floyer (2008) from the perspective of this study was that the tapered blade tip led to a much smaller zone of horizontal and forward compaction than either of the larger conical or rounded probe tips. The relative size and shape of the blade tip in the current study (and also that of Fukue, 1977) is shown in Figure 2a. Since the leading edge of the blade is blunt rather than tapered, it might be more appropriate to consider the full thickness of the blade as the scaling length, $L$, rather than half the thickness. However, in either case the length scale is comparable to or smaller than many common grain sizes encountered in seasonal snow (Fierz and others, 2009). This introduces the grain size (or a relationship between the grain size and available pore space for densification) as the dominant scaling parameter for the horizontal deformation around the tip.

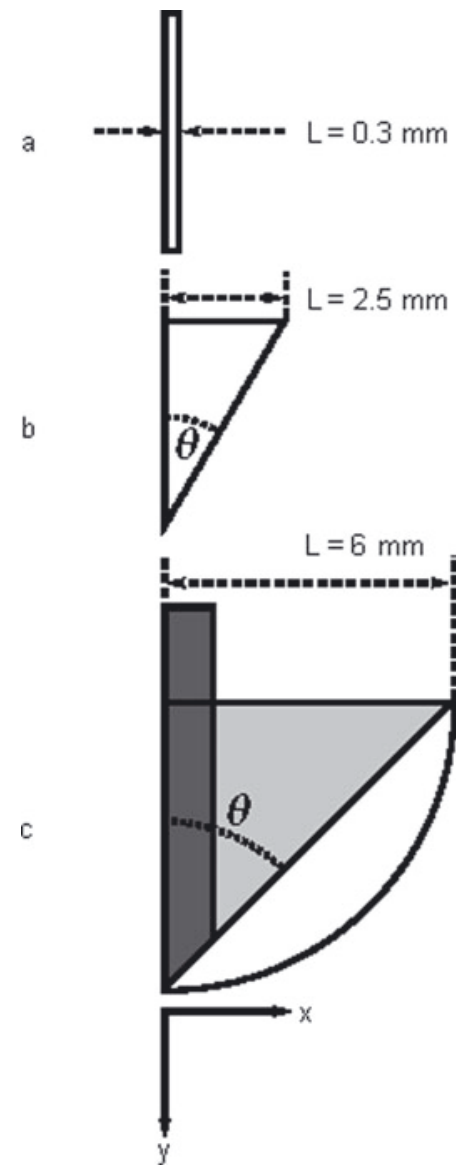

Fig. 2. Scaled representation of penetrometer tips, each in a plane of symmetry. (a) Thin blade used by Fukue (1977), with the same leading-edge dimensions as the blade in the present study. (b) SnowMicroPen (SMP) dimensions, with $\theta=30^{\circ}$. (c) Tips used by Floyer (2008), with rounded tip (unshaded) and conical tip (light gray, $\theta=45^{\circ}$ ) of the same radius. The blade tip (dark gray) had $L=1 \mathrm{~mm}, \theta \approx 45^{\circ}$.

The tip of the SMP (Fig. 2b), for comparison, has a base radius of $2.5 \mathrm{~mm}$ and a shallower cone half-angle $\left(30^{\circ}\right)$ than the conical tip used by Floyer (2008). This should lead to a relatively smaller zone of compaction around the SMP compared to the SABRE probe. The relative difference between the scaling length, $L$, for the SMP and the thin blade in the present study is about the same as the relative size difference between the tapered blade and conical tip used by Floyer (2008), though the cone and blade tip angles are different. Though the precise relative shape and size of the compaction zones for the SMP and the thin blade in this study cannot (and need not) be determined, it can be argued based on the results of Floyer (2008) and from simple dimensional scaling arguments that the thin blade will horizontally compact less snow as it penetrates than any other common hardness measure considered here.

Since the blade tip in the present study is both blunt and thin, the forward compaction may scale disproportionately with $L$. Whiteley and Dexter (1981) found that a $1 \mathrm{~mm}$ diameter probe required $\sim 50 \%$ more pressure than a $2 \mathrm{~mm}$ diameter probe to penetrate sandy soils. The explanation may lie in the development of a passive nose cone being pushed ahead of the probe, similar qualitatively to that observed by Floyer (2008). The shape and size of this nose cone does not appear to have a simple scaling relationship 
Table 1. Correlation matrix for data reported by Martinelli (1971). The upper diagonal elements contain Spearman's rank correlation coefficients, $r_{\mathrm{s}}{ }^{*}$ and the lower diagonal elements are the $p$-values. Boldface indicates statistically significant correlations at the $\alpha=0.05$ level. The lower part of the table shows the range of each variable $(n=98)$

\begin{tabular}{|c|c|c|c|c|c|}
\hline & $f_{\mathrm{t}}$ & $\rho$ & $R_{\mathrm{ram}}$ & $T$ & $E$ \\
\hline$f_{\mathrm{t}}$ & & 0.919 & 0.928 & 0.023 & 0.011 \\
\hline$\rho$ & $<0.001$ & & 0.940 & 0.013 & -0.045 \\
\hline$R_{\text {ram }}$ & $<0.001$ & $<0.001$ & & -0.054 & -0.075 \\
\hline$T$ & 0.822 & 0.899 & 0.594 & & 0.078 \\
\hline$E$ & 0.918 & 0.662 & 0.465 & 0.448 & \\
\hline \multicolumn{3}{|c|}{ Nominal tensile strength, $f_{t}$} & \multicolumn{3}{|c|}{$0.4<f_{\mathrm{t}}<246.0 \mathrm{kPa}$} \\
\hline \multicolumn{3}{|c|}{ Density, $\rho$} & \multicolumn{3}{|c|}{$68<\rho<491 \mathrm{~kg} \mathrm{~m}^{-3}$} \\
\hline \multicolumn{3}{|c|}{ Ram hardness, $R_{\text {ram }}$} & \multicolumn{3}{|c|}{$9.81<R_{\text {ram }}<2207 \mathrm{~N}$} \\
\hline \multicolumn{3}{|c|}{ Temperature, $T$} & \multicolumn{3}{|c|}{$-22.4<T<-1.4^{\circ} \mathrm{C}$} \\
\hline \multicolumn{3}{|c|}{ Grain size, $E$} & \multicolumn{3}{|c|}{$0.2<E<1.5 \mathrm{~mm}$} \\
\hline
\end{tabular}

*Spearman's rank correlations are shown rather than Pearson's productmoment correlations, $r$, for several reasons. First, Pearson's $r$ is based on the assumption of linear dependence between the two variables. However, associations among the mechanical properties of snow are often nonlinear. Pearson's $r$ also contains the assumption that the underlying parent distributions of the two variables are normal (though violations of this assumption are not severe if the sample size is large). Finally, Pearson's $r$ is much more sensitive to outliers. Spearman's $r_{\mathrm{s}}$ is a non-parametric alternative which tests for any monotonic relationship when the assumptions for using Pearson's $r$ are not met.

with the penetrometer shape and size, especially when the probe tip size is comparable to the grain size. Therefore, comparison of the forward compaction for blunt-tipped thin blades versus other penetrometers is more difficult and uncertain.

\subsection{Hardness and strength}

Bradley (1966) underlined the importance of direct strength measurements for comparison against hardness tests, though few studies have systematically done this. Comparing resistograph measurements with the compressive strength of snow columns containing weak basal layers, Bradley (1966) found that the minimum resisting stress from the resistograph roughly correlated with the compressive strength of the basal layer.

Martinelli (1971) reported data relating both ram hardness and density to centrifugal tensile strength. We analyzed these data to compare the two different proxies for strength. Both the ram hardness and density show very high (and nearly equal) correlations with the nominal centrifugal tensile strength (Table 1). The ram hardness and density are also highly correlated with each other. From these data the ram hardness appears no better (nor worse) than density for correlating with strength. Other studies attempting to relate ram hardness to strength have been largely unsuccessful (Shapiro and others, 1997).

Fukue (1977) empirically correlated blade penetration force with cohesive strength in several ways. First, artificial snow samples were allowed to sinter over time at a temperature conducive to bond growth. The unconfined compressive strength of the samples increased with age and therefore bond strength. Thin-blade penetration tests were then paired with unconfined compressive strength tests on similar snow samples undergoing sintering. The maximum blade penetration force strongly correlated with unconfined compressive strength, suggesting a link between bonding and blade penetration. Second, confined compression tests were performed at rates both below and above an identified ductile-to-brittle transition in compression rate. Following each test, a thin-blade penetration measurement was performed on the sample. Samples that had been compressed at rates above the ductile-to-brittle transition, and therefore had broken bonds, had systematically lower penetration resistance than samples that had been slowly compressed in the ductile range.

Schneebeli and Johnson (1998) directly compared centrifugal tensile strength and average penetration resistance using an early version of the SMP with a cone half-angle of $45^{\circ}$ and a $5 \mathrm{~mm}$ diameter cone tip. The tensile strength measurements showed a high amount of scatter when expressed either as a function of density or average penetration resistance. However, the uncertain repeatability of the centrifugal tensile tests may have contributed to the large scatter (Schneebeli and Johnson, 1998).

We are not aware of any other direct measurements of strength that have been compared against SMP resistance signals. Recent studies have analyzed or derived parameters from SMP signals and compared them with either the results of avalanche instability tests, which can provide indices of strength, or with published strength values. Birkeland and others (2004) found that the maximum resistance recorded by the SMP in a weak surface-hoar layer increased as a shear strength index increased. Neither the mean nor median resistance in the layer was significantly correlated with the shear strength increase. Lutz and others (2009) used resistance values and drop frequencies from the SMP to calculate a grain-scale strength index, and observed changes in this index with artificial load changes in three compression tests. Marshall and Johnson (2009) calculated theoretical values of strength from SMP signals and compared these values against tensile, compressive and shear strength values, expressed as a function of density, from the literature. Around half of the SMP-derived strength values were higher than any published values. This might be explained by the assumption in the calculations that all of the resisting force (less a small amount of friction) was due to the elastic deflection and rupture of bonds (Marshall and Johnson, 2009). Accounting for a compaction component in the resistance signal may have brought the derived strength values into better agreement with measurements.

\section{METHODS}

The blade hardness measurements were carried out in two settings. One was in excavated snow pits, alongside standard stratigraphic snow-profiling techniques used in avalanche work (CAA, 2007; Fierz and others, 2009). The second was in a cold laboratory, where the blade hardness measurements were paired with tensile strength tests on samples extracted from the natural snow cover. The force gauge, blade attachment and measurement technique are detailed first.

\subsection{Force gauge and blade attachment}

The force gauge used was a Chatillon DFE series with a full bridge strain-gauge load cell. Figure 3 shows the gauge and blade attachment. The load cell capacity was $250 \mathrm{~N}$, with a resolution of $0.1 \mathrm{~N}$. The gauge accuracy was certified to within $\pm 0.25 \%$ full scale $( \pm 0.6 \mathrm{~N})$. The gauge was 


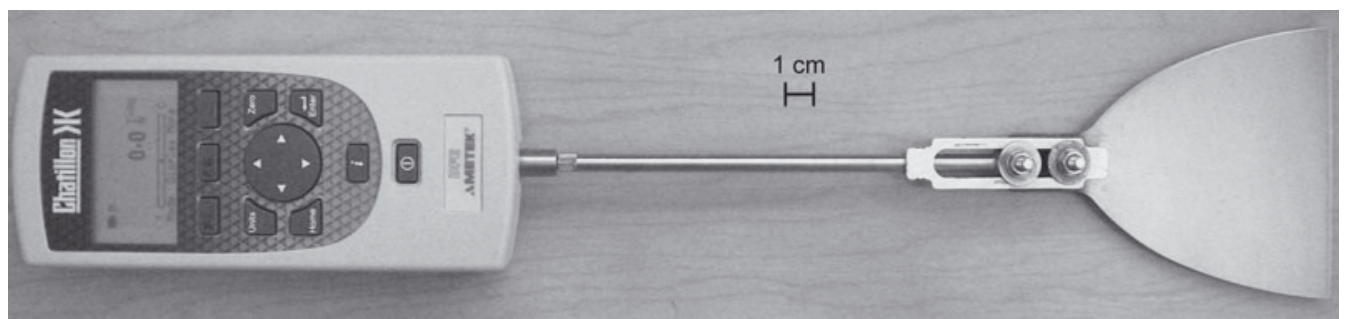

Fig. 3. Blade hardness gauge.

periodically tested for accuracy by hanging dead weights from a hook attached to it. These tests confirmed the accuracy of the gauge in the approximate range $5-95 \%$ of the full scale.

The operating temperature range of the gauge was specified as -1 to $49^{\circ} \mathrm{C}$, but the typical testing temperature was in the range -10 to $0^{\circ} \mathrm{C}$. When used in the field, the gauge was kept in an insulated container and only brought out just before use. Most tests involving the gauge lasted only a few minutes. This likely limited the actual temperature drop of the load cell in the interior of the gauge relative to the ambient temperature. In the cold laboratory, however, the gauge was exposed to cold temperatures for longer periods of time, and temperature effects might have been more significant. The specified temperature effect on zero load level was $0.09 \mathrm{~N}^{\circ} \mathrm{C}^{-1}$ relative to the calibration temperature. The median ambient temperature in the laboratory was about $-5^{\circ} \mathrm{C}$, and the calibration temperature was $23^{\circ} \mathrm{C}$, suggesting a possible shift in the zero point of the load cell of up to $2.5 \mathrm{~N}$. However, the actual internal temperature of the load cell was probably slow to change relative to the ambient temperature, buffered by the thick housing of the gauge and the internal circuitry (the gauge manufacturer used $30 \mathrm{~min}$ stabilization times when testing the load-cell performance within the range of operating temperatures). The liquid crystal display and battery life of the load cell were not affected down to air temperatures as low as $-20^{\circ} \mathrm{C}$.

The data-sampling rate of the force gauge was $5000 \mathrm{~Hz}$. It was possible to record a continuous signal at this rate, which could be sent via a cable to a data logger or a computer, or simply to record the peak force in tension or compression. For this study, only the peak resisting force was recorded. For future studies, high-resolution measurements of penetration resistance could be obtained. In particular, the variance of penetration resistance over the length scale of interest $(10 \mathrm{~cm})$ could be useful, in addition to the peak force. However, this would require a more accurate and sensitive force gauge than used in the present study.

The thin blade used was a $10 \mathrm{~cm}$ wide, $0.6 \mathrm{~mm}$ thick paint-scraper blade with a blunt leading edge. Only the leading $2 \mathrm{~mm}$ of the blade was $0.6 \mathrm{~mm}$ thick. Behind the leading edge, the blade tapered to $0.5 \mathrm{~mm}$ thickness. This thickness profile was related to a hardening finish at the tip of the blade. The handle was removed from the original paint scraper, and two bolts were used to clamp the blade to an aluminum turnbuckle (Fig. 3). One of the bolts also clamped a nut that secured the end of the threaded rod extending from the force gauge. The primary cost of the apparatus was the force gauge, as the paint scraper cost around $\$ 10$ (US\$ 2007). The digital force gauge cost around $\$ 1,250$ (US\$2007).

The leading edge of the blade extended $\sim 30 \mathrm{~cm}$ from the front of the force gauge. This distance could have been reduced by $\sim 12 \mathrm{~cm}$ by using a shorter threaded rod extending from the load cell. The combined weight of the threaded rod and blade assembly was $\sim 100 \mathrm{~g}$. The effect of this cantilevered weight did not cause a load response in the load cell. The assembled blade apparatus was also rigid torsionally, and the load cell was not sensitive to manual twisting of the blade. During penetration tests, twisting, bending or other deflection of the blade was never sensed.

\subsection{Measurement technique}

Blade hardness measurements were carried out by pushing the blade $3-5 \mathrm{~cm}$ into the surface of the snow, either into an exposed pit wall or into a snow sample in a cold laboratory, at an estimated penetration speed of $\sim 10 \mathrm{~cm} \mathrm{~s}^{-1}$ (Fig. 4). The blade was then withdrawn and the maximum force of penetration was recorded as the blade hardness index.

The adopted notation for recording the blade hardness index used the symbol ' $B$ ' (Fig. 1). This distinguished the blade hardness from the commonly used ' $R$ ' for the hand hardness or the ram hardness (Fierz and others, 2009). Unless otherwise noted, the orientation of the blade was parallel to the stratigraphic layering of the snow cover and the blade width was $10 \mathrm{~cm}$. Variations on this notation are explained as they are introduced below.

The penetration speed was high to ensure that the peak force fell into the rate-independent portion of the brittle range identified by Fukue (1977). It was hypothesized that this would maximize the consistency across observers. At this penetration speed, the $5000 \mathrm{~Hz}$ data-sampling rate of the force gauge records hundreds of samples per centimeter of penetration. This gives high confidence that during rapid penetration of the blade the true peak load was accurately captured.

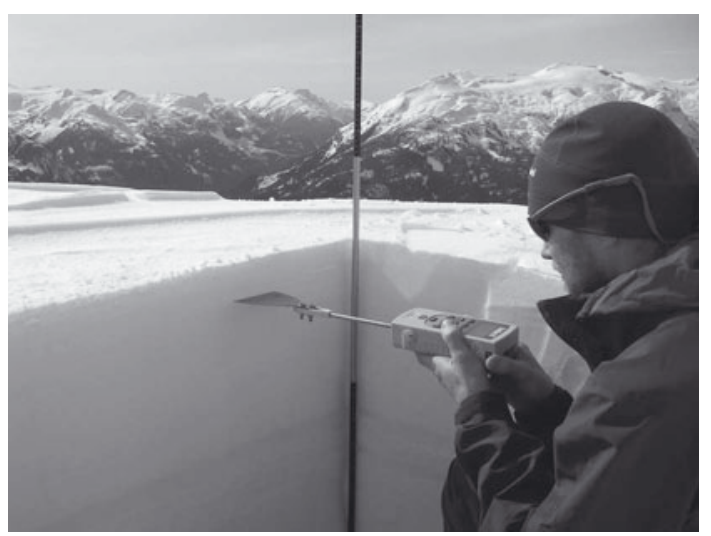

Fig. 4. Carrying out a blade hardness measurement with the blade oriented parallel to the stratigraphic layering. 


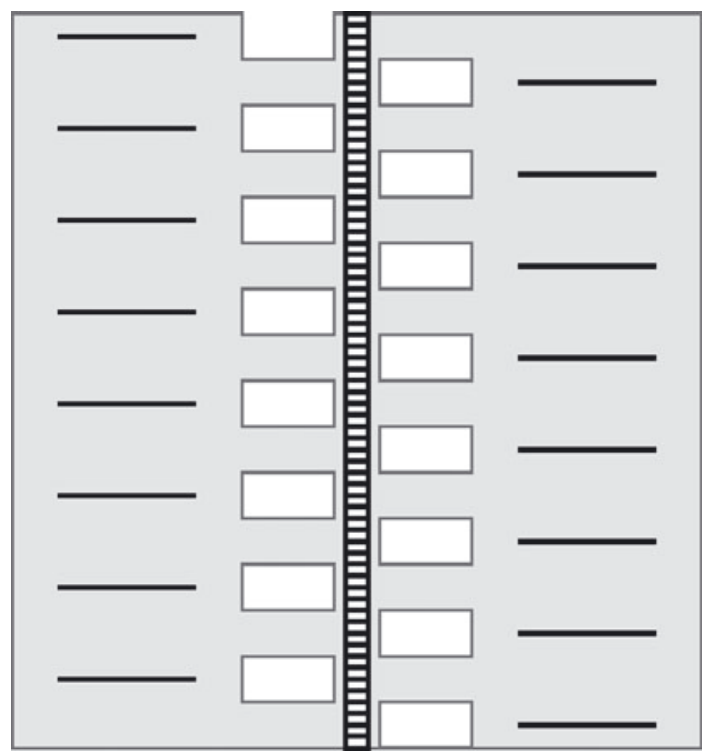

Fig. 5. Schematic of paired density and blade hardness measurements, looking at the face of an exposed snow-pit wall. The central strip represents a meter stick, the open rectangles are the holes left by the density sampling and the solid black lines are the blade hardness measurements.

\subsection{Standard stratigraphic profiling}

In an excavated snow pit, the standard profile measurements included hand hardness of identified stratigraphic layers, temperature measurements every $10 \mathrm{~cm}$ of depth from the surface to the ground, grain size and form classification by sampling snow crystals from each layer and examining them on a gridded screen under $10 \times$ magnification, density measurements and water-content characterization using a hand test. Details of these standard methods can be found in CAA (2007) and Fierz and others (2009).

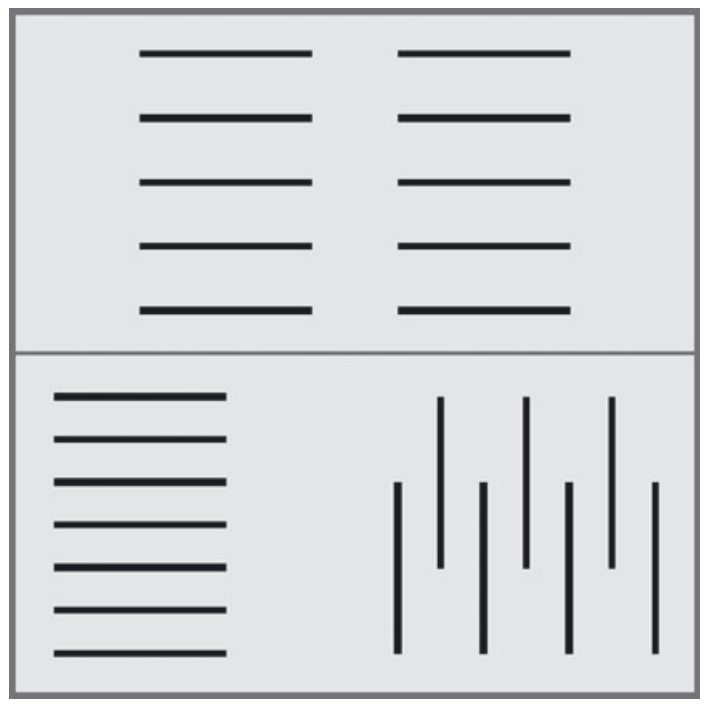

Fig. 6. Schematic of blade hardness measurement technique in two different layers, looking at the face of an exposed pit wall. Homogeneous layers are identified manually in a snow pit for this type of test grouping. In the top layer, ten measurements are shown, distributed evenly over the thickness of the layer. In the bottom layer, an equal number of slope-parallel and slope-normal measurements are shown.

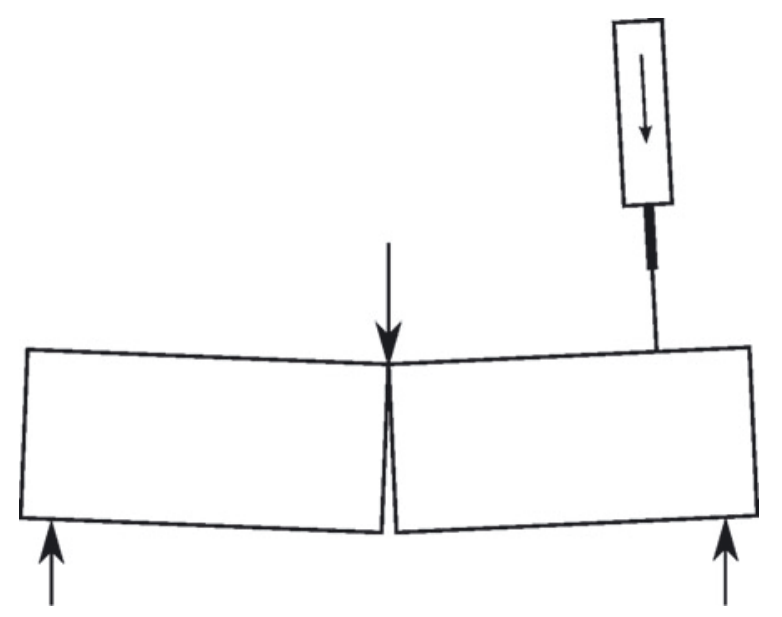

Fig. 7. Schematic of paired tests of tensile strength and blade hardness. The snow sample was first broken in three- or four-point bending (three-point bending shown). Next, a blade hardness measurement was taken (upper right) using a part of the sample that experienced low stress during the strength test.

The characterization of hand hardness in this study was consistently done using the 10-15 N force standard. Plus and minus qualifiers were used for finer-scale distinctions. For example, a hand hardness index of ' $2+$ ' was recorded as 2.3 and a ' $3{ }^{\prime}$ ' was recorded as 2.7. An approximate uncertainty was then added to reflect the imprecision and subjectivity of the test and to facilitate comparison with results from the old $50 \mathrm{~N}$ force standard. We estimated that the $50 \mathrm{~N}$ force standard would result in a hand hardness index of one level lower for characterizing the same snow. For example, if we characterized a snow layer as having hardness index 3, we reported the approximate range for comparison as $2-3$. A factor-of-five force difference, representing the maximum difference between the old and new standards, may lead to an even greater difference in reported hand hardness indices.

Blade hardness measurements added only a few minutes to standard stratigraphic profiles. Density measurements were typically paired with single blade hardness measurements as a function of depth (Fig. 5). Often groups of ten blade hardness measurements were made in manually identified homogeneous snow layers for characterizing the variability of the blade hardness index (Fig. 6). The effects of the blade orientation, width and penetration rate were also investigated in snow pits adjacent to standard profiles.

\subsection{Laboratory strength testing}

The blade hardness gauge was also used in a cold laboratory containing a universal testing machine for measuring the tensile strength of cohesive snow samples. A blade hardness measurement was paired with each strength test (Fig. 7). Dry cohesive snow samples were first extracted from homogeneous layers of at least $10 \mathrm{~cm}$ thickness in the natural snow cover. The samples were cut out using a stainless-steel rectangular cutter with a sharpened leading edge. The most common specimen size had dimensions $50 \mathrm{~cm}$ long, $10 \mathrm{~cm}$ deep and $10 \mathrm{~cm}$ wide.

Once extracted, the samples were transported to a nearby cold laboratory for testing the same day. They were weighed in the laboratory for calculation of the bulk density. They were fractured in unnotched and weight-compensated three- (or four-)point bending tests. The peak force recorded 


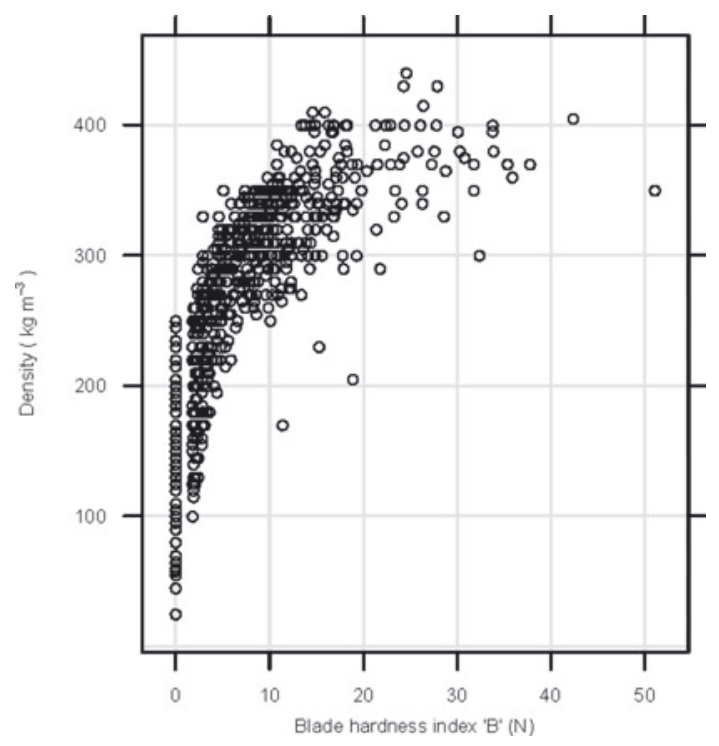

Fig. 8. Density versus blade hardness index, $B$, from 24 snow-pit profiles carried out over the winters of 2007/08 and 2008/09. The Spearman's rank correlation coefficient is $0.89, p$-value $<0.001$ $(n=628)$.

in the test was used to calculate the nominal tensile strength using Timoshenko beam theory (Timoshenko, 1940). Immediately after a strength test, a blade hardness measurement of the sample was taken, along with the temperature, grain size and grain form classification. The grain size and form were determined by examining a sample of snow crystals under a microscope on a crystal screen similar to that used in the field.

\section{RESULTS}

\subsection{Density versus blade hardness index}

For a given snow layer, which was typically characterized by a single density, there was wide scatter in blade hardness indices (Fig. 8). The coefficient of variation (COV) of repeated tests (usually ten tests) within a layer decreased with increasing layer density, and the slope was statistically significant in a linear regression $(p<0.001)$. Due to the density-hardness correlation, the COV also decreased with increasing mean blade hardness index, and this slope was also statistically significant $(p=0.02)$. Cohesionless snow, hereafter defined as snow with $B=0 \mathrm{~N}$, had no clear relation with density. Cohesionless snow was observed with densities ranging from about 30 to $250 \mathrm{~kg} \mathrm{~m}^{-3}$.

In our dataset (628 in situ test pairs), not a single blade hardness index was registered between 0.0 and $1.7 \mathrm{~N}$, indicating a gauge sensitivity problem. More than $90 \%$ of the values were $<20 \mathrm{~N}$, indicating only a small range of the full capacity $(250 \mathrm{~N})$ of the load cell was used. A total of 99 values of $B=0 \mathrm{~N}$ were recorded, many of which we interpret as legitimate negligible resistance values in cohesionless snow. The true resisting force for some of these tests was likely nonzero, however, but too low to be accurately resolved with the load cell.

\subsection{Penetration rate effects}

Penetration rate effects were considered to be the primary source of possible variability for results obtained with different operators. In one test series, pairs of fast and slow blade hardness measurements were carried out side by side. The same gauge operator was used for all tests in order to isolate the rate effect. The penetration rates were subjectively judged, with the standard $10 \mathrm{~cm} \mathrm{~s}^{-1}$ rate considered fast. For the slow tests, the penetration rate was around a few $\mathrm{cm} \mathrm{s}^{-1}$, or approximately one order of magnitude slower. These penetration speeds correspond to bond-scale strain rates on the order of $10^{-3} \mathrm{~s}^{-1}$ for the slow tests and $10^{-2} \mathrm{~s}^{-1}$ for the fast tests. Both these rates are in the rate-independent portion of the brittle range identified by Fukue (1977) (as a function of penetration rate) and Narita (1983) (as a function of strain rate).

A total of 40 pairs of tests were carried out side by side comparing one fast and one slow measurement at the same depth and within the same layer. All tests were done in a single location, with pairs of tests conducted every $3 \mathrm{~cm}$ of depth from near the surface of the snowpack to the ground. This ensured that at least one test pair was conducted within every manually identified layer. The blade was oriented slope-parallel for all tests.

In this test series, there were eight test pairs in snow of hand hardness index 1-2 in which one of the two tests (fast or slow) had a blade hardness index of $0 \mathrm{~N}$. Given the gauge sensitivity problem near $0 \mathrm{~N}$, these test pairs are discarded from the following analysis. In the 32 pairs of tests for which both the fast and slow results were nonzero, the ratio of fast to slow hardness, $B_{\text {fast }} / B_{\text {slow, }}$ had a mean and median of 1.1 . The ratio of fast to slow hardness did not consistently correlate with any other measured snow property. A Wilcoxon signed rank test was performed on the logarithm of the ratio (the logarithm symmetrizes the ratio about zero) to test whether the fast and slow results were different. The test indicated that the ratio was not significantly different from 1 at the $\alpha=0.05$ level $(p=0.07)$.

A second test series was carried out within a single homogeneous layer (hand hardness index 3-4). A total of 20 tests were carried out, 10 fast and 10 slow. For consistency of speed, the fast measurements were carried out first in a spatial cluster, followed by the slow measurements adjacent to the fast cluster. The blade was again oriented parallel to the layering.

For this test series, the mean hardness was $18.6 \mathrm{~N}$ (range 15.0-22.7) for the fast tests and $15.6 \mathrm{~N}$ (range 12.5-18.8) for the slow tests. The COVs of the fast and slow tests are the same at 0.15 . Welch's $t$ test indicated significance in the difference between the means at the $\alpha=0.05$ level $(p=0.02)$.

\subsection{Blade orientation}

Paired groups of tests were conducted to explore the effect of the orientation of the blade (Fig. 6) on the mean hardness and variability. For these tests, snow layers were first sought that were homogeneous and at least $10 \mathrm{~cm}$ thick to allow blade penetration perpendicular to the layering. In such layers, ten tests were carried out in each of two orientations. The first ten tests were carried out with the width of the blade parallel to the layering $\left(B_{\|}\right)$and the next ten were perpendicular to the layering $\left(B_{\perp}\right)$. The groups of penetration tests were carried out immediately adjacent to one another to avoid, as much as possible, encountering horizontal changes in layer properties. The mean blade hardness index for each group of ten tests $\left(\bar{B}_{\|}\right.$and $\left.\bar{B}_{\perp}\right)$ as well as the range and standard deviation were recorded. In total, 12 groups of 


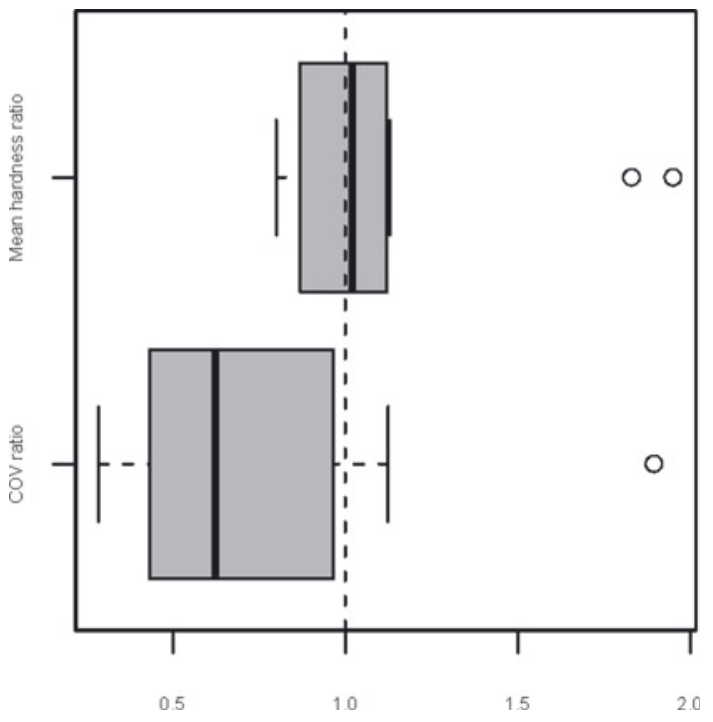

Fig. 9. Box plot showing the ratio of mean blade hardness index normal to the layering to mean hardness parallel to the layering $\left(\bar{B}_{\perp} / \bar{B}_{\|}\right)$and mean normal to parallel $\mathrm{COV}\left(\overline{\mathrm{COV}}_{\perp} / \overline{\mathrm{COV}}_{\|}\right)$. The boxes contain the inner quartile range, the whiskers extend to data points within 1.5 times the inner quartile range from the median, outliers are drawn as individual points, and the thick black line is the median. Each box plot represents 12 group means, with each group containing ten tests in each orientation. The dashed vertical line is drawn to indicate no difference between orientations.

such orientation tests were carried out in different layers. In order to compare the tests across layers with different properties, the ratios of normal to parallel mean blade hardness index, $\bar{B}_{\perp} / \bar{B}_{\|}$, and coefficient of variation, $\overline{\mathrm{COV}}_{\perp} / \overline{\mathrm{COV}}_{\|}$, were calculated for each layer tested.

The mean hardness appeared to be independent of blade orientation (Fig. 9, top). The bulk of the mean hardness ratios $\left(\bar{B}_{\perp} / \bar{B}_{\|}\right)$clustered close to 1 . The majority of layers tested showed lower variability in slope-normal than slope-parallel tests. In these cases the COV ratios, $\overline{\mathrm{COV}}_{\perp} / \overline{\mathrm{COV}}_{\|}$, were $<1$. The individual values of the COV ranged from 0.06 to 2.25 for slope-normal tests (mean 0.42, median 0.21) and from 0.13 to 2.0 for the slope-parallel tests (mean 0.35, median 0.16). A Wilcoxon signed rank test indicated that the COV ratio was different from 1 at the $\alpha=0.05$ level $(p=0.02)$.

\subsection{Blade size effect}

A wider blade was attached to the gauge in an attempt to bring out more detail than the $10 \mathrm{~cm}$ blade, especially in very soft and soft snow (hand hardness indices 1 and 2, respectively). A $20 \mathrm{~cm}$ blade with a thickness of $0.48 \mathrm{~mm}$ and a blunt leading edge (another off-the-shelf paint-scraper blade) was used for comparison. The large blade apparatus weighed $\sim 280 \mathrm{~g}$ (compared to $100 \mathrm{~g}$ for the $10 \mathrm{~cm}$ blade attachment) and had a cantilever length $\sim 3 \mathrm{~cm}$ shorter than the $10 \mathrm{~cm}$ blade. The additional cantilever weight did not induce an axial load in the load cell.

A total of 55 paired tests were carried out with the 10 and $20 \mathrm{~cm}$ wide blades. Each test pair was conducted side by side within the same layer. As with the variable penetration rate tests, test pairs were carried out every $3 \mathrm{~cm}$ of depth from the surface to the ground.

In the first ten pairs of size effect tests in very soft snow (hand hardness index 1) near the surface, both blades registered $B=0 \mathrm{~N}$. As the hardness increased with increasing depth, however, the $20 \mathrm{~cm}$ blade was the first to record values of $B>0 \mathrm{~N}$. This was the case in six pairs of tests in snow that was transitioning from hand hardness index 2 to 3 . In this snow, the $20 \mathrm{~cm}$ blade gave nonzero hardness values whereas the $10 \mathrm{~cm}$ blade did not register.

Overall, 39 of the 55 pairs of tests had nonzero hardness values for both blades. Among these pairs, the ratio of blade hardness between the 20 and $10 \mathrm{~cm}$ blades, normalized by the cross-sectional area of the blade tip $\left(0.96\right.$ and $0.6 \mathrm{~cm}^{2}$, respectively) ranged from 0.5 to 1.6 , with a mean and median of 0.9 and a standard deviation of 0.2 .

\subsection{Blade and hand hardness}

The lowest values of the blade hardness index $(B<5 \mathrm{~N})$ typically correlated with weakly cohesive snow of hand hardness index 1.7-3.7 (Fig. 9). There is considerable overlap between blade hardness indices for neighbouring hand hardness categories. The blade hardness data in Figure 9 are the group means from 52 different snow layers in which typically ten blade hardness measurements (parallel to the stratigraphic layering) were taken in each layer. The plotted hand hardness categories are those that correspond to cohesive snow as measured by $B>0 \mathrm{~N}$. The blade hardness in layers of hand hardness index 0.7-1.3 was always $0 \mathrm{~N}$. In the context of this study, such snow was considered cohesionless. In layers of hand hardness index 1.7-3, some values of $B=0 \mathrm{~N}$ were recorded, but the mean of repeated tests was always $>0$.

The variability of repeated blade hardness tests decreased with increasing hand hardness. The COV was highest in any snow that still contained decomposing and fragmented forms, which were most often found in young snow that was in the process of bond formation (and thus had low hand hardness). The next highest COV was found in faceted crystals. The lowest COVs in repeated measures were from rounded grains and mixed rounded and faceted grains.

\subsection{Blade hardness index as a proxy for strength}

Recently deposited snow layers were monitored and sampled for laboratory testing as soon as the snow was cohesive enough to extract, handle and transport. It was not possible to extract any samples characterized by hand hardness index 1 because the snow was too weak. When snow was just cohesive enough to extract, the blade hardness of the snow would, in nearly every case, register above $0 \mathrm{~N}$.

Only 9 of 238 strength tests in the laboratory were paired with a blade hardness index of $0 \mathrm{~N}$. These nine samples were from the softest and most fragile snow layer that was successfully tested in the laboratory. We suspect that the true value of blade hardness index for many (if not all) of these samples was likely 0-1.7 N. Lack of gauge sensitivity rather than lack of bond strength prevented quantifying the blade hardness index of these samples.

In our data, the tensile strength correlated much better with the blade hardness index than with the density (Table 2). The blade hardness index had a nearly equal correlation coefficient with the density as with the tensile strength. This suggests that the blade hardness index could be used to predict both density and strength, equally well, with a single measurement.

A subset of the laboratory strength tests $(n=143)$ also had precise deflection measurements at the bottom of the beam. 
Table 2. Spearman's rank correlation coefficients (upper diagonal) and $p$-values (lower diagonal) for our laboratory data. Boldface indicates statistical significance at the $\alpha=0.05$ level. The lower part of the table shows the range of each variable $(n=238)$

\begin{tabular}{|c|c|c|c|c|c|}
\hline & $f_{\mathrm{t}}$ & $\rho$ & $B$ & $T$ & $E$ \\
\hline$f_{\mathrm{t}}$ & & 0.644 & 0.844 & -0.066 & -0.325 \\
\hline$\rho$ & $<0.001$ & & 0.819 & 0.002 & 0.085 \\
\hline$B$ & $<0.001$ & $<0.001$ & & -0.046 & -0.118 \\
\hline$T$ & 0.311 & 0.972 & 0.475 & & -0.099 \\
\hline$E$ & $<0.001$ & 0.192 & 0.070 & 0.126 & \\
\hline \multicolumn{3}{|c|}{$\begin{array}{l}\text { Tensile strength, } f_{\mathrm{t}} \\
\text { Density, } \rho \\
\text { Blade hardness index, } B \\
\text { Temperature, } T \\
\text { Grain size, } E\end{array}$} & \multicolumn{3}{|c|}{$\begin{array}{c}3.4<f_{\mathrm{t}}<66.7 \mathrm{kPa} \\
149<\rho<364 \mathrm{~kg} \mathrm{~m}^{-3} \\
0.0<B<17.4 \mathrm{~N} \\
-12.0<T<0.0^{\circ} \mathrm{C} \\
0.5<E<1.0 \mathrm{~mm}\end{array}$} \\
\hline
\end{tabular}

These measurements allowed for the calculation of the flexural modulus, analogous to an elastic (or linear viscoelastic) modulus. For this subset, the Spearman correlation coefficient between the blade hardness and the flexural modulus was 0.68 and was highly significant $(p<0.001)$.

\section{DISCUSSION}

\subsection{Density and hardness}

Tables 1 and 2 have similar variables which are significantly correlated. The lower correlation between the blade hardness index and density, compared with that between ram hardness and density, may be due to several factors. The ram hardness, which deflects and compacts more snow as the cone tip penetrates, might be expected to correlate better with density than a thin-blade measure which minimizes compaction during penetration. Our dataset also contained snow with mixed rounded and faceted forms, compared with that of Martinelli (1971) who did not sample any coarse-grained lower layers in which faceted forms may have been present. At equal densities, snow with faceted forms is weaker than rounded forms (Jamieson, 1988). The smaller range of densities tested in our data may also be a factor in the lower correlation.

The wide variability in mechanical properties of snow at a given density is well known. Takeuchi and others (1998) and Höller and Fromm (2010) observed high scatter between density and flat-plate hardness measures. Keeler and Weeks (1968) and Martinelli (1971) reported increasing scatter in ram hardness with increasing density, which is consistent with the blade-hardness-density data (Fig. 8).

\subsection{Penetration rate effects}

Given the high variability in snow properties, it was not surprising that no statistically significant rate effect was observed when pairing single blade hardness tests at different penetration rates. The typical COV of repeated measures in the same layer was on the order of $0.1-1$. This high level of variability, inherent in snow properties, makes in situ testing for systematic rate dependence difficult.

In the grouped test series with ten fast and ten slow measurements within the same layer, the statistically significant rate effect observed was the opposite of what was expected. The slow tests were weaker than the fast tests,

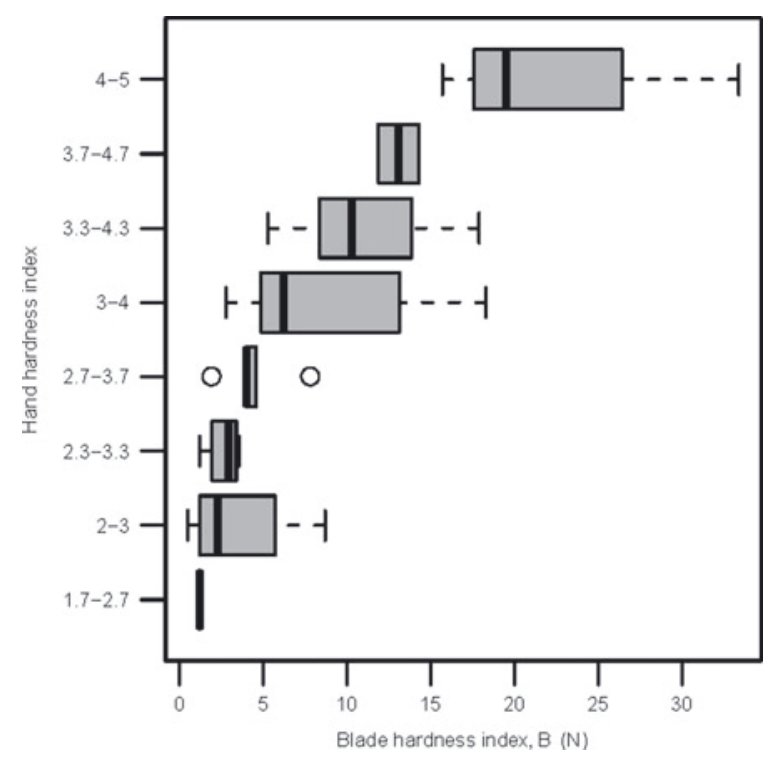

Fig. 10. Box plot of hand hardness index versus blade hardness index for cohesive snow. The boxes contain the central $50 \%$ of the data points; the whiskers extend to points within 1.5 times the inner quartile range from the median. Outliers are plotted as individual points. Overlap between hand hardness indices is related to the assumed uncertainty and imprecision of the hand test $(n=520)$.

which conflicts with the precise laboratory results of Fukue (1977) and Narita (1980). The strain rate of the fast blade hardness tests, on the order of $10^{-2} \mathrm{~s}^{-1}$, is higher than any of the previous laboratory results.

Horizontal spatial variability cannot be ruled out as a factor in these results, as the fast and slow tests were separated by up to $50 \mathrm{~cm}$ within the same layer. Takeuchi and others (1998) and Höller and Fromm (2010) demonstrated horizontal variability using push-pull hardness measures at similar length scales. Therefore it cannot be confirmed that the snow properties were the same for the two spatially separated test series. This point could have been addressed by spatially pairing fast and slow tests, alternately. The rate effects could also be influenced by a rate dependence in the development of a nose cone of grains being pushed ahead of the blade tip.

Therefore there is still some uncertainty regarding the dependence of the blade hardness index on penetration rate. Rate effects could be further investigated using a universal testing machine to drive the blade into snow samples at precise speeds. The force gauge used in this study has a mounting backplate which would facilitate integration with a testing machine. The testing machine used for the strength tests in this study had a maximum crosshead speed of $1.25 \mathrm{~cm} \mathrm{~s}^{-1}$, so it could be used to investigate the slower push speeds. Recording the penetration resistance at $5000 \mathrm{~Hz}$ in this sort of testing, rather than just the peak force, would address many of these questions. Such tests would indicate whether the schematic interpretation of the blade hardness measure (Fig. 1), based on the lower penetration speeds of Fukue (1977), is appropriate for the high push speeds used in this study.

When comparing the blade hardness results obtained by different operators, the results varied no more than would be expected given the observed variability in repeated measures using the same operator. The dataset was not 
consistently divided by users to permit formal statistical analysis to confirm this point, however. Three people (the first author and two field assistants) were the primary operators of the gauge for the data contained in this paper, and the results from each operator were taken as interchangeable.

Additional tests are necessary using different people pushing the blade hardness gauge into the same layer in order to more conclusively address the consistency across operators. However, given the commonly observed COV of repeated measures (on the order of 0.1-1) in homogeneous snow from the same operator (assuming consistent push speeds for a given operator), it is doubtful that a statistically significant difference in operator results will be found. This result is unique when compared to, for example, the hand hardness test which requires a subjective judgement about penetration force which can vary across observers.

\subsection{Blade orientation}

The lack of dependence of mean hardness on blade orientation in homogeneous layers is likely the result of the careful selection of layers that did not contain thin hard or soft sublayers or noticeable gradients in hardness or other properties from top to bottom. In the presence of stratigraphic changes in layers that were not sensed manually, conditions which were likely present in some cases, the lack of dependence on orientation probably stems from the depth averaging of the slope-parallel measurements. In many cases this technique will capture small hard or thin sublayers.

In most practical in situ applications, it made most sense to orient the blade parallel to the layering. The observation that tests conducted normal to the layering had lower variability than parallel-oriented tests is important, however. In scenarios where only a single measurement was or could be taken (e.g. in the laboratory on snow samples that did not permit multiple measurements), the blade was typically oriented perpendicular to the layering. This is effectively equivalent to saying that, given the choice between sampling from two populations with equal means but different variances, preference was given to sampling from the lower-variance population.

\subsection{Blade size effect}

When normalized by the cross-sectional area of the blade tip, the $20 \mathrm{~cm}$ blade gave slightly (but not significantly) lower values of penetration resistance. This could be related to a slightly smaller zone of compaction around the $20 \mathrm{~cm}$ blade due to its smaller thickness. The dependence of the results on the width of the blade could be explored further, though the $10 \mathrm{~cm}$ length scale was motivated by considerations from the fracture mechanics of slab avalanches. This length scale was also convenient from the perspective of ease of use, especially when compared to the $20 \mathrm{~cm}$ blade which was heavier and more awkward to align with the snow.

The original motivation for using a wider blade was to attempt to capture the transition between cohesionless and cohesive snow. It turned out that the force-gauge sensitivity problem near zero was the limiting factor in soft snow, rather than the blade width. The operating range of the gauge was very low compared to the full-scale capacity of the gauge. Rather than changing the blade width, a gauge with a lower capacity and higher sensitivity near zero will better identify the threshold penetration resistance that separates cohesive from cohesionless snow.

\subsection{Hardness and strength}

The blade hardness index characterizes an averaged measure of penetration resistance over a length scale of about 100 grains. The index compares favourably side by side with tensile strength and flexural modulus measurements in the laboratory. The tensile strength correlated higher with the blade hardness index than with any other variable in our data (Table 2). Other investigations (e.g. Martinelli, 1971) have shown similar correlations with different measures of hardness and tensile strength. The reason that density continues to be used as the primary index variable for strength and other mechanical properties of snow is related to the lack of standardization and adoption of a hardness measure across disciplines interested in snow mechanics and avalanches. The blade hardness gauge in this study is easy to use, inexpensive and appears promising as a tool for addressing this issue.

Our observation that the softest snow that we could physically handle and transport to the laboratory had the lowest $(0-2 \mathrm{~N})$ values of the blade hardness index is an independent confirmation that thin-blade penetration resistance indicates sufficient bonding between snow crystals to give strength to macroscopic volumes of snow. This is the same conclusion with regard to blade hardness and unconfined compressive strength found by Fukue (1977). We conclude that the blade hardness can be used to classify snow as cohesionless for $B \approx 0 \mathrm{~N}$, and cohesive for higher values of $B$. Future research will aim to quantify this threshold more precisely.

\subsection{Applications}

The blade hardness gauge developed in this study can be easily adopted by avalanche-forecasting and -control operations that still rely heavily on snow pit observations. Many operations also cannot afford the cost of a probe penetrometer and are disinclined to adopt technology that requires post-processing, a steep learning curve or any subjective judgements. The blade hardness gauge was designed to complement existing observation techniques rather than attempt to eliminate the need to dig a snow pit. The blade hardness is an intuitive measure, analogous to the hand hardness test which is common in avalanche operations. As a research tool, the blade hardness measure shows promise as an objective proxy for macroscopic properties of interest in avalanche applications and snow mechanics generally.

The blade hardness gauge could be used to characterize the strength of thick persistent weak layers that are commonly related to slab avalanches (McClung and Schaerer, 2006). For example, the gauge could track the relative hardness of a newly buried weak layer, and the storm snow overlying it, as they both evolve and gain (or lose) strength. It could also be used to track the loss of cohesion in snow during facet formation or as it approaches the melting temperature.

The blade hardness may also be useful for characterizing the strength of snow at higher densities, such as in firn snow. A smaller blade could be used in such a scenario because the rationale for the $10 \mathrm{~cm}$ length scale related to avalanches would not apply. This would reduce the potential for blade bending or twisting in stiffer snow. A higher-capacity force gauge would be necessary, though, and there would likely be a limiting density beyond which a blade could no longer be pushed into the snow. 


\subsection{Limitations}

In principle, it is desirable to have larger sample sizes for many of the hypothesis tests and other comparisons made in this study. Given the destructive sampling technique and the size of the blade, however, this was often not possible. The area taken up by increasing the number of tests would increase the dependence of the results on the spatial variability of snow properties, making conclusions more difficult to draw even if the hypothesis test results appeared more robust. Moreover, part of the motivation in the development of this gauge was to provide a fast, supplemental piece of information related to or dependent on snow microstructure rather than to investigate in detail the microstructure itself.

The types of snow investigated in this study were limited to what was available in the natural snow cover. The range of most properties (Table 2) is appropriate for avalanche applications. Most of the tests in this study were done in dry snow. A limited number of tests in moist snow were carried out, but not enough to test for any significant differences with dry snow. Further testing needs to be done to determine how the penetration resistance changes in moist to wet snow.

Additional work also needs to be done to relate the cohesion threshold identified in this study to the cohesion threshold at which slab avalanches first begin to occur in storm snow. Snow avalanches are reported in the hand hardness index range of 1-2 (Schweizer and Jamieson, 2001), though a large uncertainty exists in these values. The lowest hand hardness values for samples that could be handled in this study were in the range $1.7-2.7$, which suggests that some slab avalanches may occur in snow that is weakly cohesive but too weak to be handled for testing.

There is a potential boundary condition effect associated with measuring hardness in the wall of an excavated snow pit and using the results to characterize the properties of snow in situ where the stress state is different. The observations in the present study, with the COV on the order of 0.1-1 for closely spaced clusters of resistance values in homogeneous snow, suggest that measuring the influence of internal stress amid the spatial variability of natural snow is difficult. Moreover, the relative hardness of adjacent layers is often as important a piece of information as actual hardness scores in stability evaluation (e.g. Schweizer and Jamieson, 2007).

The capacity of the digital force gauge used in this study did not match the operating range, which likely contributed to the observed sensitivity problems at the bottom $1 \%$ of the scale. We did not conduct any calibration tests covering the bottom $5 \%$ of the scale, so we can only speculate as to the origin of the observed $1.7 \mathrm{~N}$ threshold penetration resistance. Temperature effects on the load cell also likely played a role. Errors introduced by temperature effects were likely larger in the laboratory results than the in situ results. As most of the data were obtained at ambient temperatures between 0 and $-10^{\circ} \mathrm{C}$, the relative shift in the zero point of the load cell across the data is small $(\sim 0.2 \mathrm{~N})$.

A load cell with a capacity in the range $30-50 \mathrm{~N}$ with at least $0.1 \mathrm{~N}$ resolution and better than $1 \%$ accuracy would be more appropriate for future investigations with a $10 \mathrm{~cm}$ blade. Additional calibration procedures should be conducted to more precisely characterize the function of the gauge at low temperature and low load. Comparing the results in the present paper against those obtained with a more appropriate load cell will be the subject of future work.

\subsection{Conclusions}

A thin-blade hardness gauge was developed that characterizes an average penetration resistance over a length scale appropriate for characterization of snow properties relevant to avalanches. Horizontal compaction of snow around the blade is minimized relative to all other common hardness measures. The gauge is an inexpensive, small and lightweight tool that can be used in the field or laboratory with results that are objective and consistent across observers. The measurement technique is simple and adds little time to other experimental methods. Compared with other standard measurements (e.g. density, temperature and grain size), the blade hardness index is the best variable for correlating with snow strength, one of the most important properties in the fracture mechanics of slab avalanches and slope stability.

\section{ACKNOWLEDGEMENTS}

We are grateful for the support of the Natural Sciences and Engineering Research Council of Canada, Canadian Mountain Holidays, and the University of British Columbia. Two anonymous reviewers provided valuable comments that helped improve an early manuscript.

\section{REFERENCES}

Bader, H., R. Haefeli, E. Bucher, J. Neher, O. Eckel and C. Thams. 1954. Snow and its metamorphism. SIPRE Transl. 14.

Bažant, Z.P., G. Zi and D.M. McClung. 2003. Size effect law and fracture mechanics of the triggering of dry slab snow avalanches. J. Geophys. Res., 108(B2), 2119-2229.

Birkeland, K., K. Kronholm, M. Schneebeli and C. Pielmeier. 2004. Changes in the shear strength and micro-penetration hardness of a buried surface-hoar layer. Ann. Glaciol., 38, 223-228.

Bradley, C.C. 1966. The snow resistograph and slab avalanche investigations. IASH Publ. 69 (Symposium at Davos 1965 Scientific Aspects of Snow and Ice Avalanches), 251-260.

Bradley, C.C. 1968. The resistograph and the compressive strength of snow. J. Glaciol., 7(51), 499-506.

Camponovo, C. and J. Schweizer. 2001. Rheological measurements of the viscoelastic properties of snow. Ann. Glaciol., 32, 44-50.

Canadian Avalanche Association (CAA). 2007. Observation guidelines and recording standards for weather, snowpack, and avalanches. Revelstoke, B.C., Canadian Avalanche Association.

Colbeck, S.C. and 7 others. 1990. The international classification for seasonal snow on the ground. Wallingford, Oxon, International Association of Scientific Hydrology. International Commission on Snow and Ice.

Conger, S.M. and D.M. McClung. 2009. Comparison of density cutters for snow profile observations. J. Glaciol., 55(189), 163-169.

De Quervain, M. 1951. Strength properties of a snow cover and its measurement. SIPRE Transl. 9.

Fierz, C. and 8 others. 2009. The international classification for seasonal snow on the ground. Paris, UNESCO-International Hydrologic Programme. (IHP Technical Documents in Hydrology 83.)

Floyer, J.A. 2008. Layer detection and snowpack stratigraphy characterisation from digital penetrometer signals. (PhD thesis, University of Calgary.)

Floyer, J.A. and J.B. Jamieson. 2010. Rate-effect experiments on round-tipped penetrometer insertion into uniform snow. J. Glaciol., 56(198), 664-672.

Fukue, M. 1977. Mechanical performance of snow under loading. (PhD thesis, McGill University.) 
Höller, P. and R. Fromm. 2010. Quantification of the hand hardness test. Ann. Glaciol., 51(54), 39-44.

Jamieson, J.B. 1988. In situ tensile strength of snow in relation to slab avalanches. (MSc thesis, University of Calgary.)

Johnson, J.B. and M. Schneebeli. 1999. Characterizing the microstructural and micromechanical properties of snow. Cold Reg. Sci. Technol., 30(1-3), 91-100.

Keeler, C.M. and W.F. Weeks. 1968. Investigations into the mechanical properties of alpine snow-packs. J. Glaciol., 7(50), 253-271.

Lutz, E., K.W. Birkeland and H.P. Marshall. 2009. Quantifying changes in weak layer microstructure associated with artificial load changes. Cold Reg. Sci. Technol., 59(2-3), 202-209.

Mackenzie, R. and W. Payten. 2002. A portable, variable-speed penetrometer for snow pit evaluation. In Stevens, J.R., ed. Proceedings of the International Snow Science Workshop, 29 September-4 October 2002, Penticton, British Columbia. Victoria, B.C., British Columbia Ministry of Transportation. Snow Avalanche Programs, 294-300.

Marshall, H.P. and J.B. Johnson. 2009. Accurate inversion of highresolution snow penetrometer signals for microstructural and micromechanical properties. J. Geophys. Res., 114(F4), F04016. (10.1029/2009JF001269.)

Martinelli, M., Jr. 1971. Physical properties of alpine snow as related to weather and avalanche conditions. USDA Forest Serv. Res. Pap. RM-64.

McClung, D.M. 2007a. Dry snow slab shear fracture speeds. Geophys. Res. Lett., 34(10), L10502. (10.1029/2007GL029261.)

McClung, D.M. 2007b. Fracture energy applicable to dry snow slab avalanche release. Geophys. Res. Lett., 34(2), L02503. (10.1029/ 2006GL028238.)

McClung, D.M. and P. Schaerer. 2006. The avalanche handbook. Third edition. Seattle, WA, The Mountaineers.

Nakamura, T., O. Abe, R. Hashimoto and T. Ohta. 2010. A dynamic method to measure the shear strength of snow. J. Glaciol., 56(196), 333-338.
Narita, H. 1980. Mechanical behaviour and structure of snow under uniaxial tensile stress. J. Glaciol., 26(94), 275-282.

Narita, H. 1983. An experimental study on tensile fracture of snow. Contrib. Inst. Low Temp. Sci., Ser. A 32, 1-37.

Pielmeier, C. and M. Schneebeli. 2003. Stratigraphy and changes in hardness of snow measured by hand, rammsonde and snow micro penetrometer; a comparison with planar sections. Cold Reg. Sci. Technol., 37(3), 393-405.

Schneebeli, M. and J.B. Johnson. 1998. A constant-speed penetrometer for high-resolution snow stratigraphy. Ann. Glaciol., 26 107-111.

Schweizer, J. and J.B. Jamieson. 2001. Snow cover properties for skier triggering of avalanches. Cold Reg. Sci. Technol., 33(2-3), 207-221.

Schweizer, J. and J.B. Jamieson. 2007. A threshold sum approach to stability evaluation of manual snow profiles. Cold Reg. Sci. Technol., 47(1-2), 50-59.

Schweizer, J., J.B. Jamieson and M. Schneebeli. 2003. Snow avalanche formation. Rev. Geophys., 41(4), 1016. (10.1029/ 2002RG000123.)

Shapiro, L.H., J.B. Johnson, M. Sturm and G.L. Blaisdell. 1997. Snow mechanics: review of the state of knowledge and applications. CRREL Rep. 97-3.

Sigrist, C., J. Schweizer, H. Schindler and J. Dual. 2005. On size and shape effects in snow fracture toughness measurements. Cold Reg. Sci. Technol., 43(1-2), 24-35.

Szabo, D. and M. Schneebeli. 2007. Subsecond sintering of ice. Appl. Phys. Lett., 90(15), 151916. (10.1063/1.2721391.)

Takeuchi, Y., Y. Nohguchi, K. Kawashima and K. Izumi. 1998. Measurement of snow-hardness distribution. Ann. Glaciol., 26 $27-30$.

Timoshenko, S. 1940. Strength of materials, Part 1. Elementary theory and problems. New York, D. Van Nostrand Co.

Whiteley, G.M. and A.R. Dexter. 1981. The dependence of soil penetrometer pressure on penetrometer size. J. Agr. Eng. Res., 26, 467-476.

MS received 29 June 2010 and accepted in revised form 28 January 2011 